\title{
Possible role of free radicals generated by pseudohypoxia in the regulation of hepatic glucose output. An in vitro model using rat liver microsomal glucose 6-phosphatase
}

\author{
I. Wittmann, I.Mazák, L. Wagner, J. Nagy \\ Second Department of Medicine, University Medical School of Pécs, Pécs, Hungary
}

Summary Hepatic glucose output is decreased by hyperglycaemia through an unknown mechanism. We hypothesize that free radicals generated by hyperglycaemic pseudohypoxia might cause glucose output to decrease by inhibiting glucose 6-phosphatase - a key enzyme of gluconeogenesis. To prove this a model experiment was performed on a microsome fraction of rat liver. One of the characteristic features of pseudohypoxia due to hyperglycaemia is an increase in the ratio of $\mathrm{NADH} / \mathrm{NAD}^{+}$, so in the present study the changes in NADH - induced glucose 6-phosphatase activity were investigated as related to the release of inorganic phosphate (Pi) derived from glucose 6phosphate. After incubation for $50 \mathrm{~min}$, Pi release was significantly reduced by NADH $(4.026 \pm 0.189$ vs $2.696 \pm 0.429 \mu \mathrm{mol} \cdot \mathrm{l}^{-1} \cdot \mathrm{mg}$ protein ${ }^{-1}$, control vs NADH samples, $p<0.01$ ). The decrease in the activity of glucose 6-phosphatase generated by NADH was prevented by using desferrioxamine, an irreversible ferric chelator, butylated hydroxytoluene and Trolox, two agents which inhibit lipid peroxidation, and reduced glutathione, a non-specific radical scavanger. Superoxide dismutase, catalase and the hydroxyl radical scavenger dimethyl sulphoxide proved to be ineffective. When the above investigations were carried out in the presence of a ferric-EDTA complex the inhibition of glucose 6-phosphatase was found to be inducible by hydrogen peroxide and/or hydroxyl free radicals. These investigations seem to indicate that pseudohypoxia due to hyperglycaemia can inhibit the activity of glucose 6-phosphatase both by lipid peroxidation and by inducing hydrogen peroxide and/or hydroxyl free radicals and thus it may play a part in the glucose-induced decrease of hepatic glucose output. [Diabetologia (1997) 40: 1251-1254]

Keywords Free radical, hepatic glucose output, glucose 6-phosphatase
The inhibition of hepatic glucose output (HGO) by hyperglycaemia is a well-known phenomenon [1]. Recently it has been noticed that this phenomenon is caused by glucose-mediated autoregulation rather than by an effect of insulin. The direct glucose 6-

Received: 28 January 1997 and in final revised form: 1 July 1997

Corresponding author: Dr. I. Wittmann, Second Department of Medicine, University Medical School of Pécs, Pécs, Pacsirta u. 1. H-7624 Hungary

Abbreviations: BHT, Butylated hydroxytoluene; DMSO, dimethyl sulphoxide; G6Pase, glucose 6-phosphatase; GSH, glutathione (reduced form); $\mathrm{HGO}$, hepatic glucose output; $\mathrm{Pi}$, inorganic phosphate; SOD, superoxide dismutase. phosphatase (G6Pase) inhibitory effect of hyperglycaemia has also been demonstrated by using the hyperglycaemic clamp method [2]. The inhibition of G6Pase might explain the autoregulation of $\mathrm{HGO}$ since the key enzyme of glucose output is G6Pase whose activity enables the release of glucose from the hepatic cells. A possible means of inhibition of G6Pase induced by hyperglycaemia is by inhibition by a metabolic intermediate. However, the investigation of as many as 25 glucose metabolites did not reveal one that could have inhibited the G6Pase at physiological or pathophysiological concentrations [3]. The activity of the enzyme can also be inhibited by lipid peroxidation [4]. There is evidence indicating that hyperglycaemia alters the intracellular redox status. The NADH/NAD ${ }^{+}$ratio increases, a phenomenon 
referred to as hyperglycaemic pseudohypoxia because the same redox alteration can also be observed in hypoxic cells. Both hypoxia and pseudohypoxia can also be characterised by inducing free radical processes [5]. The increase in NADH/NAD ${ }^{+}$ratio can also be observed in the cytoplasm of hepatic cells of hyperglycaemic rats (blood glucose level $30 \mathrm{mmol} / \mathrm{l}$ ) [6].

The probability of the development of disorders associated with free radicals is also supported by the fact that parallel to the accumulation of the electron donor NADH in diabetes, disorders of iron metabolism can also be present [7]. Iron, which is capable of receiving and releasing electrons, may trigger lipid peroxidation and superoxide free radical production in this redox cycle [4]. Based on the above, it was hypothesized that by increasing the NADH concentration, elevated formation of free radicals would also occur and through free radical processes, hyperglycaemia may inhibit the activity of G6Pase and hence HGO. The present study was undertaken to provide evidence for this possibility:

Hyperglycaemia $\rightarrow \mathrm{NADH} \uparrow \rightarrow$ free radicals $\uparrow \rightarrow$ G6Pase activity $\downarrow \rightarrow \mathrm{HGO} \downarrow$

An in vitro model was developed in which G6Pase activity of microsome fractions isolated from rat liver was assayed both in the presence and absence of NADH and iron complexes. For the identification of the nature of free radicals produced, SOD (a dismutating enzyme for superoxide free radicals), catalase (a decomposing enzyme of hydrogen peroxide), desferrioxamine (an irreversible iron chelator), BHT and Trolox (inhibitors of lipid peroxidation), ethanol and dimethylsulphoxide (DMSO) (scavangers of hydroxyl free radical) as well as GSH (a non-specific scavanger) were administered.

\section{Materials and methods}

All reagents were of the highest grade available. The final concentrations used were $10 \mathrm{mmol} / \mathrm{l}$ G6Pase, $2600 \mathrm{U} / \mathrm{ml}$ catalase, $49 \mathrm{U} / 1 \mathrm{SOD}, 2 \mathrm{mmol} / 1$ desferrioxamine, $1.4 \mathrm{mmol} / \mathrm{l} \mathrm{NADH}$, $50 \mathrm{mmol} / \mathrm{l}$ DMSO, $100 \mathrm{mmol} / \mathrm{l}$ ethanol, $2 \mathrm{mmol} / \mathrm{l} \mathrm{GSH}$, $0.1 \mathrm{mmol} / 1 \mathrm{BHT}, 0.1 \mathrm{mmol} / 1$ Trolox.

Liver microsomes were isolated from male Sprague-Dawley rats weighing about 200 to $250 \mathrm{~g}$. The livers were initially perfused with $0.9 \% \mathrm{NaCl}$ and rapidly removed and routinely homogenized in a solution containing $0.25 \mathrm{~mol} / 1$ sucrose, $0.01 \mathrm{~mol} / 1$ Tris- $\mathrm{HCl}$ (pH 7.4), $0.001 \mathrm{~mol} / 1$ EDTA, $50 \mu \mathrm{mol} / 1 \mathrm{des}-$ ferrioxamine. The microsomes were isolated by differential centrifugation, washed twice with $0.125 \mathrm{~mol} / \mathrm{l} \mathrm{KCl}$ in the absence of EDTA at $100000 \mathrm{~g}$, and resuspended in $0.125 \mathrm{~mol} / \mathrm{l}$ $\mathrm{KCl}$ and stored at $-75^{\circ} \mathrm{C}$.

The ferric complexes were prepared by dissolving ferric ammonium sulfate in $0.1 \mathrm{~mol} / 1 \mathrm{HCl}$, and then diluting with the respective chelator to the appropriate stock concentration. Ferric-ATP was utilized as a 1:20 complex, whereas ferricEDTA was utilized as a 1:2 complex.
Isolated microsomes were incubated with or without $50 \mathrm{mmol} / \mathrm{l} \mathrm{Tris-HCl}$ ( $\mathrm{pH} \mathrm{7.4)} \mathrm{buffer} \mathrm{with} 10 \mathrm{mmol} / 1$ G6Pase in the presence or absence of $1.4 \mathrm{mmol} / 1 \mathrm{NADH}, 100 \mu \mathrm{mol} / \mathrm{l}$ iron complexes and scavangers over a $10-60 \mathrm{~min}$ time period at $37^{\circ} \mathrm{C}$. Control incubations with all of the reagents but without microsomes showed no NADH oxidation measuring at 340 $\mathrm{nm}$ (the concentration of the remaining NADH, in four independent measurements in ferric-ATP + Tris- $\mathrm{HCl}$ medium after $60 \mathrm{~min}$ was $98.8 \pm 4.0 \%$ (mean $\pm \mathrm{SD}$ ), in Tris- $\mathrm{HCl}$, without ferric-ATP $102.1 \pm 4.8 \%$, and in ferric-EDTA, without Tris$\mathrm{HCl}$ at $\mathrm{pH} 7.4,98.7 \pm 2.2 \%$ ). The reaction was stopped by addition of $0.5 \mathrm{ml}$ of trichloroacetic acid (10\% final concentration). The samples were centrifuged at $4^{\circ} \mathrm{C}$ for $14 \mathrm{~min}$. We used $1 \mathrm{ml}$ of the supernatant for the determination of inorganic phosphate using the assay described by LeBel et al. [8]. Protein was determined by the method of Lowry et al. [9].

Statistical analysis. All results are from three experiments carried out in duplicate. Values refer to mean \pm SD. Statistical analysis was carried out using one way repeated measures ANOVA of the SigmaStat for Windows, Version 1.0.

\section{Results}

Figure 1 illustrates Pi release as a measure of G6Pase enzyme activity with respect to time. By the end of the incubation period the enzyme activity was significantly reduced by the presence of NADH $(30 \mathrm{~min}$, $p<0.03 ; 40 \mathrm{~min}, p<0.02,50 \mathrm{~min}, p<0.01$, control vs $\mathrm{NADH}$ ). Pi release was also significantly reduced by ferric-ATP + NADH $(30,40,50 \mathrm{~min} p<0.01$, ferricATP vs ferric-ATP + NADH). Ferric-ATP + NADH, however, did not significantly lower G6Pase activity more than NADH did in the absence of ferric-ATP (at each stage $p>0.05$ ).

When the incubation was carried out in the presence of ferric-EDTA, no inhibitory effect by NADH was observed. When Tris buffer, which is thought to have scavenger activity, was not used especially with respect to hydroxyl free radical, and $\mathrm{pH}$ was set at 7.4 by $\mathrm{NaOH}$, a $36 \%$ inhibition by NADH could be observed during min 60 . $(0.178 \pm 0.012$ vs $0.131 \pm$ $0.04 \mu \mathrm{mol} \cdot \mathrm{l}^{-1} \cdot \mathrm{mg}$ protein ${ }^{-1} \cdot \mathrm{min}^{-1} \mathrm{Pi}$, ferric-EDTA vs ferric-EDTA + NADH, $p<0.01$, data not shown).

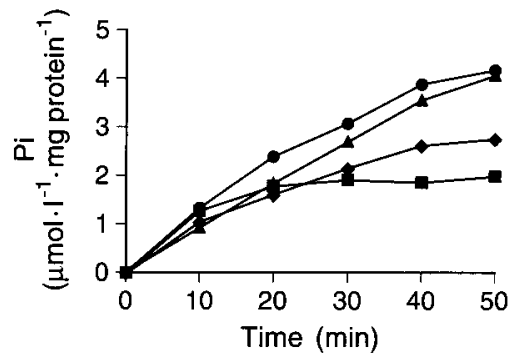

Fig.1. Inhibition of the activity of microsomal G6Pase by NADH and by ferric- ATP + NADH. Pi, Inorganic phosphate released by the enzyme from G6Pase, $\boldsymbol{\Lambda}$ control, ferricATP, NADH, $\square$ ferric-ATP + NADH. (30 min, $p<0.03$, $40 \mathrm{~min}, p<0.02,50 \mathrm{~min}, p<0.01$; control vs $\mathrm{NADH}$ and 30 , $40,50 \mathrm{~min}, p<0.01$; ferric-ATP vs ferric-ATP $+\mathrm{NADH}$ ) 


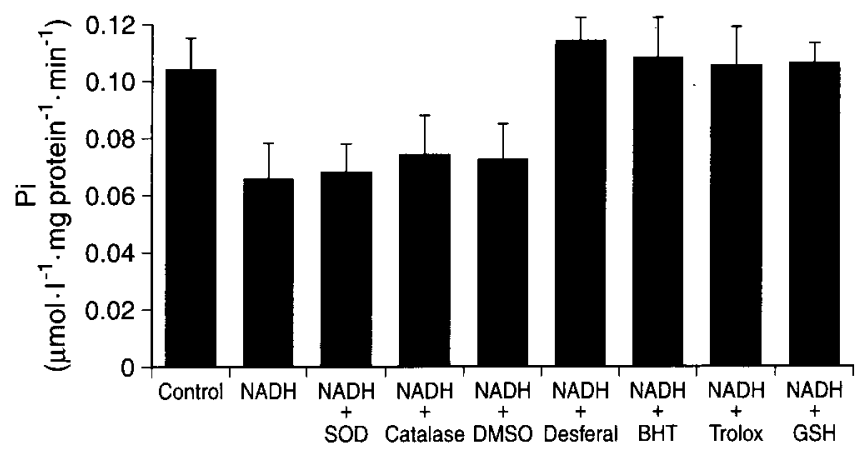

Fig. 2. Effect of scavengers on the inhibition of G6Pase enzyme evoked by NADH. Pi, Inorganic phosphate released by the enzyme from G6Pase, SOD, superoxide dismutase; DMSO, dimethyl sulphoxide; Desferal, desferrioxamine; BHT, butylated hydroxytoluene; GSH, reduced glutathione. $(p<0.01$ control vs NADH, NADH vs NADH + Desferal, $\mathrm{NADH}+\mathrm{BHT}, \mathrm{NADH}+$ Trolox, $\mathrm{NADH}+\mathrm{GSH})$

In order to analyse the potential free radical processes underlying G6Pase inhibition caused by $\mathrm{NADH}$, scavengers were added to the Tris buffer incubation medium. The results are demonstrated in Figure 2. NADH inhibition was not influenced by SOD, catalase or DMSO.

However, the inhibitory effect of NADH was completely prevented by desferrioxamine, BHT, Trolox and GSH.

When NADH inhibition was studied in the presence of ferric-EDTA, SOD and BHT proved to be ineffective in preventing loss of G6Pase activity $\left(0.131 \pm 0.004\right.$ vs $0.130 \pm 0.004 \mu \mathrm{mol} \cdot \mathrm{I}^{-1} \cdot \mathrm{mg}$ protein $^{-1}$ $\cdot \mathrm{min}^{-1} \mathrm{Pi}, \mathrm{NADH}$ vs $\mathrm{NADH}+\mathrm{SOD}, 0.131 \pm 0.004$ vs $0.139 \pm 0.011 \mu \mathrm{mol} \cdot \mathrm{l}^{-1} \cdot \mathrm{mg} \operatorname{protein}^{-1} \cdot \mathrm{min}^{-1} \mathrm{Pi}$, $\mathrm{NADH}$ vs $\mathrm{NADH}+\mathrm{BHT}, p>0.05)$. However, radical scavengers, such as catalase $(0.131 \pm 0.004$ vs $0.169 \pm 0.014 \mu \mathrm{mol} \cdot \mathrm{l}^{-1} \cdot \mathrm{mg} \operatorname{protein}^{-1} \cdot \mathrm{min}^{-1} \mathrm{Pi}$, $\mathrm{NADH}$ vs $\mathrm{NADH}+$ catalase, $p<0.01$ ), DMSO $\left(0.131 \pm 0.004\right.$ vs $0.152 \pm 0.001 \mu \mathrm{mol} \cdot \mathrm{l}^{-1} \cdot \mathrm{mg}$ protein $^{-1} \cdot \min ^{-1} \mathrm{Pi}, \mathrm{NADH}$ vs $\mathrm{NADH}+\mathrm{DMSO}$, $p<0.01)$, ethanol $(0.131 \pm 0.004$ vs $0.164 \pm 0.003 \mu \mathrm{mol}$ $\cdot \mathrm{l}^{-1} \cdot \mathrm{mg}$ protein ${ }^{-1} \cdot \mathrm{min}^{-1} \mathrm{Pi}, \mathrm{NADH}$ vs NADH + ethanol, $p<0.01)$, and GSH $(0.131 \pm 0.004$ vs $0.178 \pm$ $0.016 \mu \mathrm{mol} \cdot \mathrm{l}^{-1} \cdot \mathrm{mg}$ protein ${ }^{-1} \cdot \min ^{-1} \mathrm{Pi}, \mathrm{NADH}$ vs $\mathrm{NADH}+\mathrm{GSH}, p<0.01$ ) were found to have a protective effect against the decrease in enzyme activity in ferric-EDTA medium (data not shown).

\section{Discussion}

Our results suggest that under conditions imitating hyperglycaemia (an increase in NADH) the activity of G6Pase decreases. The degree of inhibition (26$33 \%$ ) shows a quantitative equivalence with the data obtained using in vivo hyperglycaemic clamp methodology (26\%) [2]. Using exogenous ferric-ATP, no significant increase in the inhibitory effect by
NADH was observed. This indicates that for the induction of the inhibitory effect, the presence of iron in trace amounts, mobilized by NADH from the microsomes containing ferritin [4], and partially originating from the buffers used in these experiments, is sufficient. The intracellular presence of this trace amount of mobilized iron is an essential component of our hypothesis, which may model the hyperglycaemia-induced pseudohypoxic processes. Small amounts of iron are known to remain in the microsome even after preparation in the presence of desferrioxamine [4]. The inhibition of G6Pase in the NADH medium is probably induced by lipid peroxidation since SOD, catalase and DMSO (which remove superoxide, hydrogen peroxide and hydroxyl free radical) proved to be ineffective, while BHT, Trolox and GSH were found to be effective in protecting against inhibition.

When G6Pase activity was studied in the presence of ferric-EDTA complex (a powerful microsomal hydroxyl free radical generating system), no changes were found in the presence of NADH, due to the hydroxyl free radical scavanger properties of Tris-HCL buffer. In the absence of Tris buffer a decrease in the ezyme activity was observed in the presence of $\mathrm{NADH}$, which was probably due to hydrogen peroxide and/or hydroxyl free radicals, since the decrease was catalase-sensitive, DMSO and ethanol reduced the damage to G6Pase, whereas SOD and BHT were found to be ineffective.

The three inhibitory factors of HGO are hyperglycaemia, insulin and parasympathetic stimulation [1]. The latter reflects acetylcholine signal transduction, the second messenger of which is nitric oxide. Nitric oxide may also play a role in the development of insulin action [10]. Nitric oxide is capable of binding with superoxide free radical with high affinity. It can be assumed that in our experiments hydrogen peroxide was produced through the dismutation of superoxide free radicals in the ferric-EDTA medium. As a result of the nitric oxide plus superoxide reaction peroxynitrite is produced, which, unlike nitric oxide, is incapable of activating guanylate cyclase and thus the agonist effect does not develop. Thus, parasympathetic and insulin-mediated HGO inhibition may be influenced by previous hyperglycaemic conditions which by inducing the production of free radicals, might cause disinhibition. As a result of these processes, normal, decreased or increased HGO levels can be detected in diabetic patients at any given moment. To test this in vivo, we plan to perform experiments using nitric oxide synthase substrate L-arginine and free radical scavangers in human.

We conclude that the decrease of HGO induced by hyperglycaemia can be caused by lipid peroxydation and/or by hydroxyl free radical due to an increase in NADH resulting in G6Pase inhibition. 
Acknowledgements. We are grateful to Professor A.I.Cederbaum (Department of Biochemistry, Mount Sinai School of Medicine, New York, USA) for his useful criticism and comments. This work was supported by the Hungarian National Research Foundation (OTKA 13 353), by the Foundation of the Hungarian Ministry of Welfare (T 06 699/93) and by Novo Nordisk.

\section{References}

1. Ferrannini E, DeFronzo RA (1992) Insulin actions in vivo: glucose metabolism. In: Alberti KGMM, DeFronzo RA, Keen $\mathrm{H}$, Zimmet $\mathrm{P}$ (eds) International textbook of diabetes mellitus. Wiley, Chichester NewYork Brisbane Toronto Singapore, pp 409-438

2. Guignot L, Mithieux G (1996) Hyperglycaemia, but not hyperinsulinemia, inhibits liver glucose-6-phosphatase activity in rat. Diabetologia 39: 13 (Abstract)

3. Robbins BL, Foster JD, Nordlie RC (1991) Metabolic intermediates as potential regulators of glucose-6-phosphatase. Life Sci 48: 1075-1081
4. Puntarulo S, Cederbaum AI (1994) Ferritin-dependent inactivation of microsomal glucose- 6-phosphatase. Biochim Biophys Acta 1200: 41-47

5. Williamson JR, Chang K, Frangos M et al. (1993) Hyperglycaemic pseudohypoxia and diabetic complications. Diabetes 42: 801-813

6. Williamson DH, Lund P, Krebs HA (1967) The redox state of free nicotinamide-adenine dinucleotide in the cytoplasm and mitochondria of rat liver. Biochem J 103: 514-526

7. Wolff SP (1993) Diabetes mellitus and free radicals. Free radicals, transition metals and oxidative stress in the etiology of diabetes mellitus and complications. Brit Med Bull 49: 642-652

8. LeBel D, Poirier GG, Beaudoin AR (1978) A convenient method for the ATPase assay. Anal Biochem 85: 86-89

9. Lowry OH, Rosebrough NJ, Farr AL, Randall RJ (1951) Protein measurements with the folin phenol reagent. J Biol Chem 193: 265-275

10. Wittmann I, Nagy J (1996) Are insulin resistance and atherosclerosis the consequences of oxidative stress? Diabetologia 39: 1002-1003 (Letter) 